\title{
Sequence, symmetry, and magnetic fluctuations of the magnetization reversal in exchange-biased multilayers
}

\author{
A. Paul,* E. Kentzinger, U. Rücker, D. E. Bürgler, and P. Grünberg \\ Institut für Festkörperforschung, Forschungszentrum Jülich GmbH, D-52425 Jülich, Germany
}

(Received 2 April 2004; published 10 December 2004)

\begin{abstract}
The magnetization reversal in $\left[\mathrm{Ir}_{20} \mathrm{Mn}_{80} / \mathrm{Co}_{80} \mathrm{Fe}_{20}\right]_{10}$ exchange-biased multilayers is studied by specular reflection and off-specular scattering of polarized neutrons. The reversal proceeds sequentially starting with the bottom (top) CoFe layer for decreasing (increasing) field due to the evolution of the grain size along the stack. Each CoFe layer remagnetizes symmetrically for both field branches in a nonuniform mode. Concomitant in-plane magnetization fluctuations revealed by off-specular spin-flip scattering indicate a more complex reversal mechanism than hitherto considered.
\end{abstract}

DOI: 10.1103/PhysRevB.70.224410

PACS number(s): 75.70.Cn, 61.12.Ha, 75.60.Jk

When a ferromagnet (FM) in contact with an antiferromagnet (AF) is cooled below the blocking temperature of the AF in an external field $H_{\mathrm{FC}},{ }^{1}$ a direct exchange coupling between the FM and AF layer gives rise to an unidirectional magnetic anisotropy called exchange bias. ${ }^{2}$ Consequently, the hysteresis loop of the FM layer is shifted to negative fields. Although the exchange bias effect is not yet fully understood, it is widely employed in magnetoresistive sensors ${ }^{3}$ and, thus, has recently attracted intense attention (for reviews see Refs. 4 and 5).

Asymmetric hysteresis loops due to asymmetric magnetization reversal processes are observed in many experiments, ${ }^{6-10}$ including the very first observation of exchange bias in Ref. 2. The underlying mechanism is expected to have crucial importance to elucidate the exchange bias effect. Recently, asymmetric magnetization reversal processes of $\mathrm{Fe}$ films exchange-biased by twinned $\mathrm{MnF}_{2}$ and $\mathrm{FeF}_{2}$ AF layers ${ }^{10}$ and $\mathrm{Co} / \mathrm{CoO}$ bilayers ${ }^{6,7}$ have been examined by polarized neutron reflectometry (PNR). In these reports, reversal by magnetization rotation is identified by a significant increase of the specular reflectivities in the spinflip (SF) channels $\left(R_{+-}\right.$and $\left.R_{-+}\right)$, which are exclusively of magnetic origin and correspond to in-plane magnetization components perpendicular to the guiding field $H_{\mathrm{a}}$ applied collinear to $H_{\mathrm{FC}}$. Reversal by domain nucleation and propagation does not provide enhanced SF intensities, because the magnetization is always collinear to $H_{\mathrm{a}}$ and solely shows up in the specular non-spin-flip (NSF) reflectivities $\left(R_{++}\right.$and $\left.R_{--}\right)$as an additional contribution to the nonmagnetic reflectivity. In Ref. 10 it is argued that the unidirectional anisotropy hinders the formation of domains with magnetization antiparallel to the cooling field direction and favors magnetization rotation for the decreasing field branch. ${ }^{1}$ However, when the field is increased, domains with the magnetization parallel to $H_{\mathrm{FC}}$ are energetically favorable, and the remagnetization proceeds via domain nucleation and propagation. Just the opposite reversal mechanisms are reported for the $\mathrm{Co} / \mathrm{CoO}$ system, ${ }^{6,7}$ where domain wall motion occurs for the decreasing field branch and magnetization rotation for increasing fields. Both systems are epitaxially grown, but the dependence on the direction of $H_{\mathrm{FC}}$ with respect to the twinning axes in Ref. 10 and training effects in Ref. 7 hamper a direct comparison.
In the present article, we investigate the evolution of the layer-by-layer magnetization configuration of polycrystalline IrMn/CoFe multilayers (ML's) along a full magnetization loop by specular and off-specular PNR. We find a sequential switching of the layers that we relate to the microstructural evolution along the stack. The reversal proceeds for both loop branches in a nonuniform mode and is accompanied by fluctuations of the in-plane magnetization component perpendicular to $H_{\mathrm{a}}$.

We study exchange-biased polycrystalline $\left[\mathrm{Ir}_{20} \mathrm{Mn}_{80}(6.0 \mathrm{~nm}) / \mathrm{Co}_{80} \mathrm{Fe}_{20}(3.0 \mathrm{~nm})\right]_{N}$ ML's with varying number $N$ of $\mathrm{AF} / \mathrm{FM}$ bilayers prepared by dc magnetron sputtering. We employ a 10-nm-thick $\mathrm{NiFe}$ buffer layer grown on oxidized $\mathrm{Si}$ wafers in order to improve the texture of the ML's. Prior to the measurements, the specimens are annealed for $60 \mathrm{~min}$ at $533 \mathrm{~K}$, i.e., above the IrMn Néel temperature of $520 \mathrm{~K}$, and then field-cooled to room temperature (RT) in an external field of $H_{\mathrm{FC}}=130$ Oe. Data concerning magnetic properties are taken after several remagnetization cycles in order to exclude training effects.

PNR measurements are performed at the polarized neutron reflectometer with polarization analysis $\mathrm{HADAS}^{11-13}$ at the Jülich research reactor FRJ-2 (DIDO). The neutron wavelength is fixed at $\lambda=4.52 \AA$. The instrument is equipped with a two-dimensional (2D) detector with a special spin analyzer that covers the whole detector area and thus allows simultaneously measuring specular and off-specular intensities with polarization analysis. The polarization efficiencies of the polarizer and analyzer are $96 \%$ and $95 \%$, respectively. The specimens are kept at RT and a guiding field $H_{\mathrm{a}}$ of up to $15 \mathrm{kOe}$ can be applied. We perform layer-resolved magnetometry ${ }^{14,15}$ by analyzing and fitting specular NSF and SF reflectivity cross sections. In specular geometry (angle of incidence $\alpha_{\mathrm{i}}$ equal to the exit angle $\alpha_{\mathrm{f}}$ ), the reflectivities follow from energy and in-plane momentum conservation laws. Normal wave vector transfers $Q_{\perp}=(2 \pi / \lambda)\left[\sin \left(\alpha_{\mathrm{i}}\right)\right.$ $\left.+\sin \left(\alpha_{\mathrm{f}}\right)\right]$ are probed. Off-specular scattering arises when the in-plane translational symmetry is broken by interface roughness or magnetic domains on a length scale shorter than the in-plane projection $l_{\|} \approx 2 \pi / \delta Q_{\|}$of the neutron coherence length, where $\delta Q_{\|}$is the uncertainty of the in-plane momentum transfer $Q_{\|}=(2 \pi / \lambda)\left[\cos \left(\alpha_{\mathrm{f}}\right)-\cos \left(\alpha_{\mathrm{i}}\right)\right] .{ }^{16}$ For our instru- 

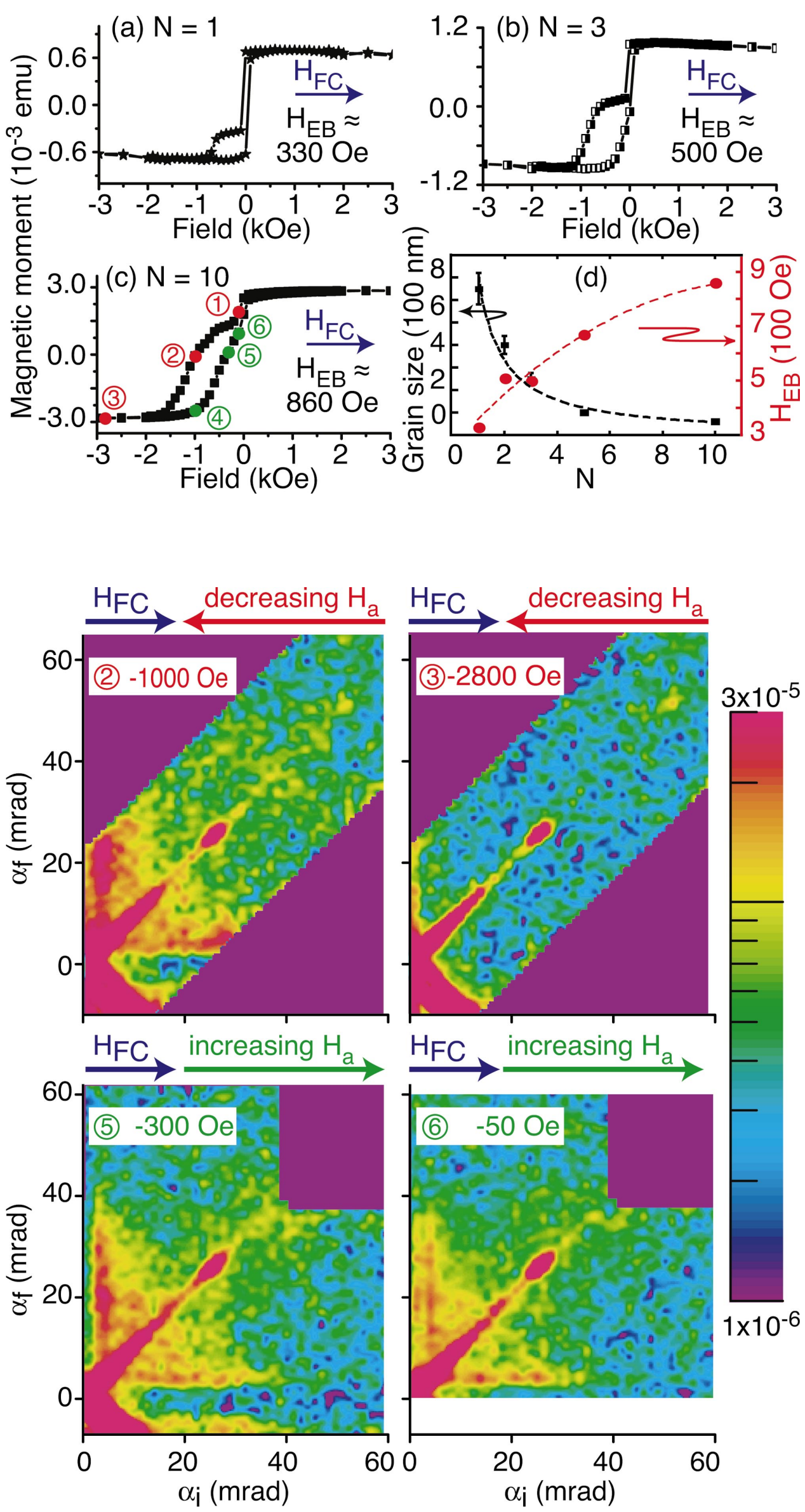

FIG. 1. (Color) (a)-(c) SQUID magnetization loops of $\mathrm{SiO}_{2} /$ $\mathrm{NiFe}(10.0 \mathrm{~nm}) /[\operatorname{IrMn}(6.0 \mathrm{~nm}) /$ $\mathrm{CoFe}(3.0 \mathrm{~nm})]_{N}$ ML's with different numbers of bilayers $N$. The relative contribution of the $\mathrm{NiFe}$ buffer decreases and $H_{\mathrm{EB}}$ increases with $N$. Circled numbers refer to Figs. 2 and 3. (d) $N$ dependences of $H_{\mathrm{EB}}$ (red) and the grain size at the surface as determined from AFM images (black).
FIG. 2. (Color) SF reflectivity maps $R_{+-}$of a $\mathrm{SiO}_{2} / \mathrm{NiFe}$ $(10.0 \mathrm{~nm}) /[\mathrm{IrMn}(6.0 \mathrm{~nm}) / \mathrm{CoFe}$ $(3.0 \mathrm{~nm})]_{10}$ ML at different positions along the magnetization loop [see Fig. 1(c)]. Off-specular intensity appears near the switching for both decreasing (panel 2) and increasing (panels 5 and 6) loop branches but is absent in the saturated state (panel 3 ). 

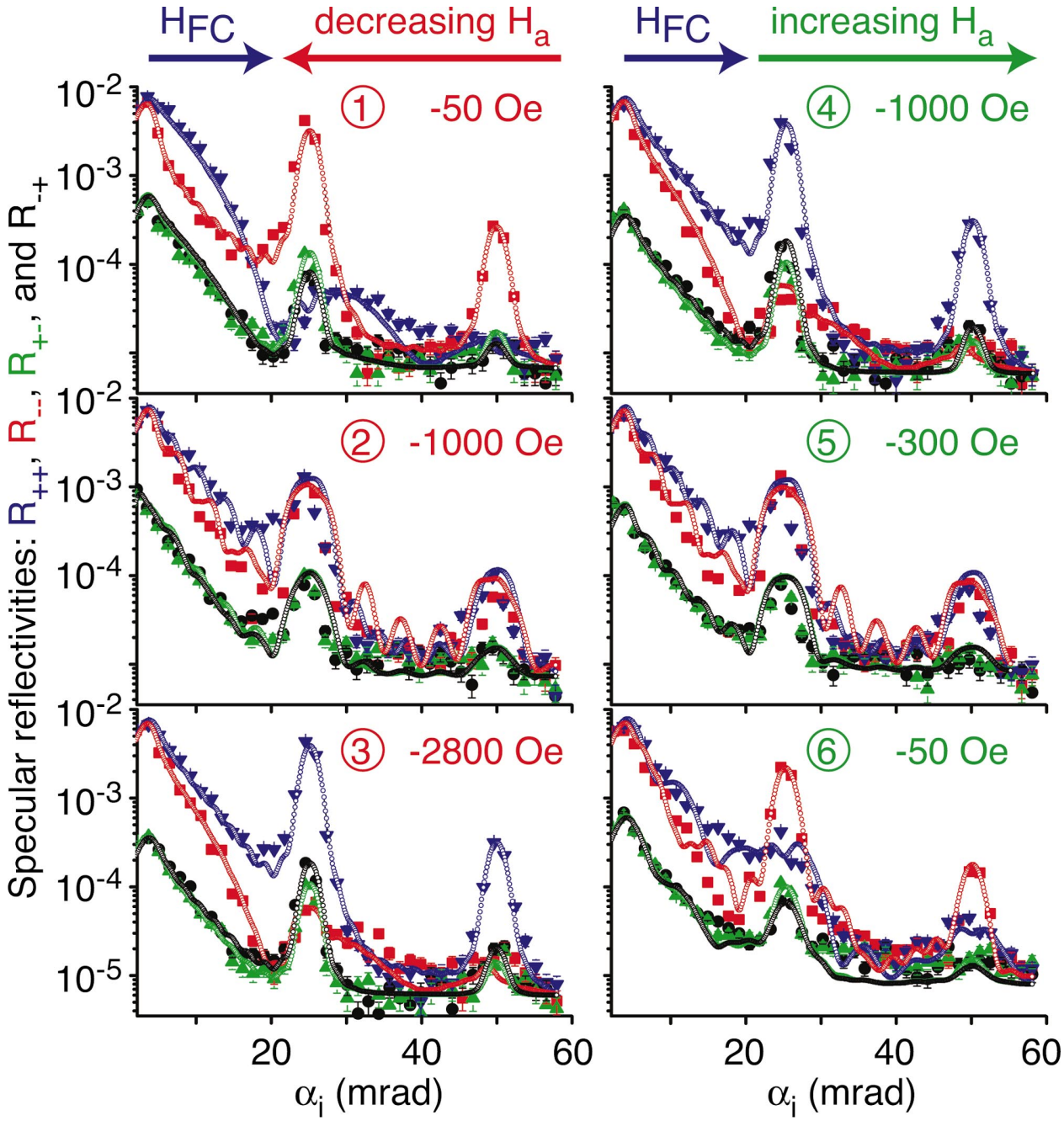

FIG. 3. (Color) Specular reflectivities $R_{++}$(blue), $R_{--}$(red), $R_{+-}$(green), and $R_{-+}$(black) of a $\mathrm{SiO}_{2} / \mathrm{NiFe}(10.0 \mathrm{~nm}) /[\operatorname{IrMn}(6.0$ $\mathrm{nm}) / \mathrm{CoFe}(3.0 \mathrm{~nm})]_{10} \mathrm{ML}$ at different positions along the magnetization loop [circled numbers refer to Fig. 1(c)]. Filled symbols are the data and open symbols the fits. ment $l_{\|}$exceeds $20 \mu \mathrm{m}$, but the resolution of the 2D detector defines an upper limit of about 20-30 $\mu \mathrm{m}$ for the resolvable lateral structure size. A lower limit of about $1 \mu \mathrm{m}$ results from the limited neutron flux at the sample position.

The microstructure and the layer quality are investigated by $\mathrm{X}$-ray reflectivity (XRR) and $\mathrm{x}$-ray diffuse scattering (XDS) measurements as well as atomic and magnetic force microscopy (AFM, MFM) imaging. ${ }^{17}$ Magnetization loops are recorded by means of a superconducting quantum interference device (SQUID) as well as the magneto-optic Kerr effect (MOKE).

Figures 1(a)-1(c) show SQUID magnetization loops for $N=1,3$, and 10 . There are always two hysteresis subloops, the narrow one corresponding to the magnetically soft $\mathrm{NiFe}$ buffer layer and the wider to the CoFe layer(s) in the ML. The relative contributions to the sample's total moment confirm this assignment. The loop shift $H_{\mathrm{EB}}$ increases from 330 Oe for $N=1$ to about 900 Oe for $N=10$ [Fig. 1(d)], while the loop shape evolves from square to slanted. We obtain similar results from MOKE measurements.

In order to understand the reason for this strong $N$ dependence we perform microstructural characterization by XRR, XDS, and AFM. ${ }^{17}$ The fitting of specular and diffuse $\mathrm{X}$-ray scattering patterns ${ }^{18}$ reveals only a rather weak increase of the interface roughness $\sigma$ from 0.3 to $0.6 \mathrm{~nm}$ and no signifi- cant dependence of the fractal dimension $h \approx 1$ and the lateral correlation length $\xi=10 \pm 5 \mathrm{~nm}$ on $N$. The vertical correlation length is larger than the total ML thickness for all $N$. Such an evolution of the microstructure along multilayered structures is quite common, ${ }^{19}$ but cannot explain the strong enhancement of $\mathrm{H}_{\mathrm{EB}} \cdot{ }^{20}$ However, AFM images reveal a strong variation of the grain size of the topmost layer from $650 \mathrm{~nm}$ for $N=1$ to $60 \mathrm{~nm}$ for $N=10$ [Fig. 1(d)], and MFM micrographs indicate a change of the FM domain structure with $N$, from extended domains for $N=1$ to structures of about $500 \mathrm{~nm}$ in diameter for $N=10 .{ }^{17}$ Therefore, we attribute the enhancement of $H_{\mathrm{EB}}$ to the shrinking of the grain size and explain it in the framework of the domain-state model for exchange bias: ${ }^{21,22}$ Grain boundaries are energetically preferred sites for domain walls in the AF. ${ }^{23}$ Smaller AF grains are accompanied by an increased density of AF domains and hence an increased excess magnetization in the AF which increases $H_{\mathrm{EB}} \cdot{ }^{22}$ For ML's with $N$ of the order of 10 , a gradual variation of the grain size and $H_{\mathrm{EB}}$ along the growth direction and, hence, a nontrivial magnetic reversal behavior is expected.

We perform PNR measurements at 15 different fields $H_{\mathrm{a}}$ applied collinear to $H_{\mathrm{FC}}$ on both sides of the hysteresis loop for a ML with $N=10$ in order to study the layer-resolved magnetization state and reversal behavior. Figure 2 shows 
the SF intensity $\left(R_{+-}\right)$maps as a function of $\alpha_{\mathrm{i}}$ and $\alpha_{\mathrm{f}}$ at four different representative fields $H_{\mathrm{a}}$ [see circled numbers in Fig. 1(c)]. The specular intensity along the line $\alpha_{\mathrm{i}}=\alpha_{\mathrm{f}}$ shows first-order and weak second-order Bragg peaks at $\alpha_{\mathrm{i}, \mathrm{f}} \approx 25$ and $50 \mathrm{mrad}$ corresponding to the bilayer thickness. The fitting of the specular reflectivities described below confirms that all these Bragg peaks are of NSF nature and appear in the SF channels due to the nonideal polarization efficiencies.

Off-specular intensity appears near the critical angle $\alpha_{\mathrm{c}}$ $\approx 4 \mathrm{mrad}$ in panels 2,5 , and 6 , i.e., in both loop branches near the reversal, but not in the saturated state at $H_{\mathrm{a}}=$ $-2.8 \mathrm{kOe}$ in panel 3. This is a clear difference to the data in Ref. 7 and indicates that the off-specular intensity is related to the reversal process rather than to magnetic disorder due to, for instance, interface roughness. The off-specular reflectivity is most intense at the two fields where the net magnetization of the ML vanishes (panels 2 and 5) and gradually diminishes (e.g., panel 6) to disappear in saturation (panel 3).

The presence of off-specular intensity confirms that the in-plane magnetization component perpendicular to the guiding field, $M_{\perp}$, is laterally inhomogeneous on a length scale smaller than $l_{\|}$. The fact that the off-specular intensity extends to $\alpha_{\mathrm{i}}=\alpha_{\mathrm{c}}$ and $\alpha_{\mathrm{f}}=\alpha_{\mathrm{c}}$ implies that the inhomogeneities occur on a length scale clearly below $1 \mu \mathrm{m}$. The high intensity at $\alpha_{\mathrm{c}}$ arises from the enhanced transmission coefficient at the critical angle and is not related to a length scale. Thus, we think of the inhomogeneities as fluctuations of $M_{\perp}$ rather than magnetic domains. These fluctuations are directly linked to the magnetization reversal of the exchange-biased system. A quantitative evaluation of the lateral length scale is beyond the scope of the present measurement geometry. Anyway, the length scale of the fluctuations is of the order of magnitude of the grain size. The absence of off-specular intensity in the Bragg sheets signifies that there is no vertical correlation of the $M_{\perp}$ fluctuations.

All four specular reflectivity channels are measured at 15 different $H_{\mathrm{a}}$, six of which are shown in Fig. 3 [circled numbers refer to Fig. 1(c)] together with least-square fits based on an extension of the Paratt formalism ${ }^{24}$ to magnetic ML's. ${ }^{25,26}$ The two peaks in the NSF channels $\left(R_{++}\right.$and $\left.R_{--}\right)$ are the first- and second-order Bragg reflections of the ML. The corresponding weak peaks in the SF channels $\left(R_{+-}\right.$and $R_{-+}$) can be reproduced in the fits by taking into account the polarization efficiencies of our setup. $R_{++}$and $R_{--}$are almost equal at $H_{\mathrm{a}}=-1000 \mathrm{Oe}$ (panel 2) on the decreasing and at $H_{\mathrm{a}}=-300 \mathrm{Oe}$ (panel 5) on the increasing branch and signify that the reversal for both loop branches proceeds via a state with an almost vanishing magnetization component collinear to $H_{\mathrm{a}}$. For all other fields $R_{++}$or $R_{--}$dominates and reflects a net magnetization collinear with $H_{\mathrm{a}}$, while the SF intensities are always much weaker. In Fig. 4(a) we show the measured SF intensity for $\alpha_{\mathrm{i}, \mathrm{f}}=\alpha_{\mathrm{c}}$ as a function of $H_{\mathrm{a}}$ and compare with the calculated SF intensity for the situation where the full ML magnetizations is aligned perpendicular to $H_{\mathrm{a}}$ (dashed line). Obviously, the data indicate a much smaller $M_{\perp}$. In a next step, we calculate the SF intensity under the assumption that the magnetization of only one CoFe layer is perpendicular to $H_{\mathrm{a}}$ and all others collinear to $H_{\mathrm{a}}$. The resulting SF intensity depends on which layer along the stack we choose to be perpendicularly magnetized. The range of val-

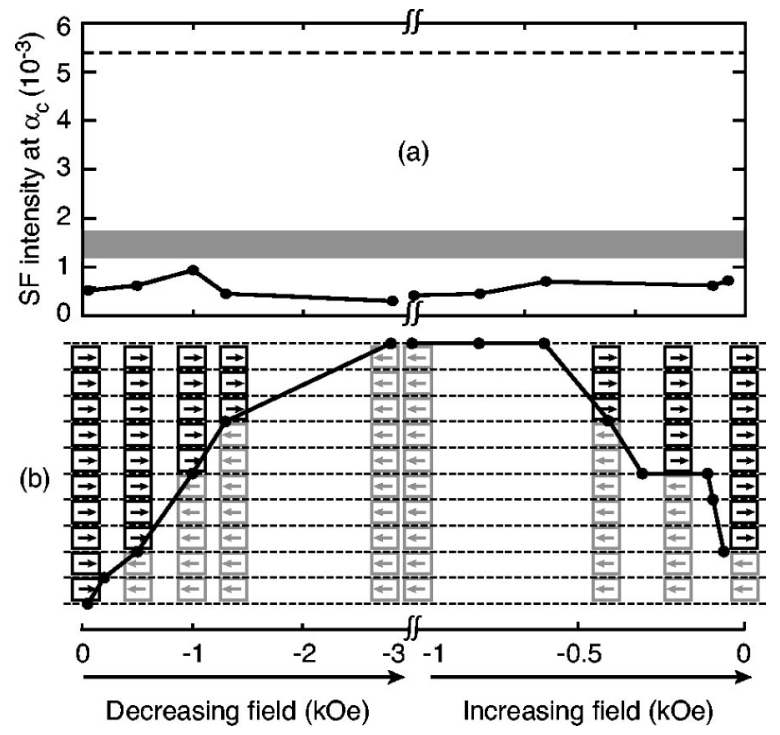

FIG. 4. (a) Experimental (symbols) and simulated (dashed line and gray region) SF intensity for $\alpha_{\mathrm{i}, \mathrm{f}}=\alpha_{\mathrm{c}}$ as a function of $H_{\mathrm{a}}$. The data are not compatible with perpendicular alignment of the whole ML (dashed line) nor a single CoFe layer (gray region); see text. (b) Switching sequence along the ML from the bottom to top and back as obtained from the fits. For some field values, each $\mathrm{CoFe}$ layer in the ML is represented by a box and the arrows indicate a layer magnetization parallel (black) or antiparallel (gray) to $H_{\mathrm{FC}}$.

ues is marked in Fig. 4(a) by the gray region. We conclude from these comparisons that the magnetization reversals in both directions do not proceed via magnetization rotation, neither of all layers together nor of one at a time. This symmetric, but nonuniform reversal mode corresponds to the situation described by Beckmann et al. ${ }^{27}$ for no misalignment between the exchange bias axis and $H_{\mathrm{a}}$. These authors considered this an experimentally unlikely situation. The observed fluctuations of $M_{\perp}$, however, reflect the instability that occurs when the effective field, that acts on the FM, is aligned with $H_{\mathrm{a}}{ }^{27}$

For a more quantitative analysis, we first fit the specular intensities in the saturated state (panel 3 in Fig. 3) to adjust the nuclear and magnetic scattering length densities, the layer thicknesses, and the interface roughness. We find excellent agreement with the XRR data for the thicknesses and the roughness and keep them as well as the nuclear scattering length densities fixed for all subsequent fits. For the nonsaturated states we have to vary the magnetization configuration of the ML, which-in principle-requires to introduce a magnetization amplitude $M_{\mathrm{i}}$ and direction $\theta_{\mathrm{i}}$ for each $\mathrm{CoFe}$ layer $(i=1, \ldots, 10)$. Based on the conclusions from Fig. 4(a) we can keep the number of fitting parameters manageable by only considering deviations from the purely collinear, single domain configurations (i.e., $\theta_{\mathrm{i}}=0^{\circ}$ or $180^{\circ}$ ) described by the mean magnetization amplitude $\left\langle M_{\mathrm{i}}\right\rangle$ and the mean angular deviation from the collinear alignment $\left\langle\Delta \theta_{\mathrm{i}}\right\rangle$. We fit the data for all permutations of collinear configurations in order to identify the configuration yielding the best agreement. The results are shown in Fig. 4(b): For decreasing $H_{\mathrm{a}}$ the CoFe layers switch sequentially from the bottom to the top, and for increasing $H_{\mathrm{a}}$ the reversal proceeds in the opposite direction. 
This sequential switching of the layers is due to the structural evolution along the ML. The layers at the top consist of smaller grains that yield a stronger exchange bias in agreement with the data in Fig. 1. Therefore, they align last with a field applied antiparallel to $H_{\mathrm{FC}}$, but first when $H_{\mathrm{a}}$ is again increased. The fitted $\left\langle M_{\mathrm{i}}\right\rangle$ does not show significant variations except for the lowest fields $(50 \mathrm{Oe})$ where it is decreased by $11 \%$ most likely due to a small number of domains that form when one approaches $H_{\mathrm{a}}=0 .\left\langle\Delta \theta_{\mathrm{i}}\right\rangle$ is small except for $-1 \mathrm{kOe}$ (point 2) on the decreasing branch, where it amounts to $8^{\circ}$. This deviation is in agreement with the maximum specular and off-specular SF intensity in Figs. 4(a) and 2 respectively.
In conclusion, we have studied in detail the remagnetization behavior of an exchange-biased multilayer. The independent, but sequential reversal of the FM layers is related to the evolution of the grain size along the stack and can be explained by extending the domain-state model $^{22}$ from a diluted to a polycrystalline antiferromagnet. The reversal of each FM layer proceeds symmetrically via a nonuniform mode for both remagnetization directions and is observed to be accompanied by fluctuations of the in-plane magnetization on a length scale comparable with the grain size. Therefore, the observed reversal mode is more complex than hitherto considered and involves processes beyond coherent rotation and simple domain wall motions.
*Author to whom correspondence should be addressed. Electronic address: A.Paul@fz-juelich.de

${ }^{1} \mathrm{We}$ assume a positive direction of $H_{\mathrm{FC}}$ and call the field sweep from positive to negative (negative to positive) saturation the decreasing (increasing) loop branch.

${ }^{2}$ W. H. Meiklejohn and C. P. Bean, Phys. Rev. 102, 1413 (1956).

${ }^{3}$ B. Dieny, J. Magn. Magn. Mater. 136, 335 (1994).

${ }^{4}$ J. Nogués and I. K. Schuller, J. Magn. Magn. Mater. 192, 203 (1999).

${ }^{5}$ A. E. Berkowitz and K. Takano, J. Magn. Magn. Mater. 200, 552 (1999).

${ }^{6}$ M. Gierlings, M. J. Prandolini, H. Fritzsche, M. Gruyters, and D. Riegel, Phys. Rev. B 65, 092407 (2002).

${ }^{7}$ F. Radu, M. Etzkorn, R. Siebrecht, T. Schmitte, K. Westerholt, and H. Zabel, Phys. Rev. B 67, 134409 (2003).

${ }^{8}$ V. I. Nikitenko, V. S. Gornakov, A. J. Shapiro, R. D. Shull, K. Liu, S. M. Zhou, and C. L. Chien, Phys. Rev. Lett. 84, 765 (2000).

${ }^{9}$ W.-T. Lee, S. G. E. te Velthuis, G. P. Felcher, F. Klose, T. Gredig, and E. D. Dahlberg, Phys. Rev. B 65, 224417 (2002).

${ }^{10}$ M. R. Fitzsimmons, P. Yashar, C. Leighton, I. K. Schuller, J. Nogués, C. F. Majkrzak, and J. A. Dura, Phys. Rev. Lett. 84, 3986 (2000).

${ }^{11}$ U. Rücker, B. Alefeld, W. Bergs, E. Kentzinger, and Th. Brückel, Physica B 276-278, 95 (2000).

${ }^{12}$ U. Rücker, W. Bergs, B. Alefeld, E. Kentzinger, and Th. Brückel, Physica B 297, 140 (2001).
${ }^{13}$ For information on HADAS see: http://www.fz-juelich.de/iff/ wns_hadas

${ }^{14}$ J. A. C. Bland, J. Vac. Sci. Technol. A 15, 1759 (1997).

${ }^{15}$ C. H. Marrows, S. Langridge, and B. J. Hickey, Phys. Rev. B 62, 11340 (2000).

${ }^{16}$ V. Lauter-Pasyuk, H. J. Lauter, B. P. Toperverg, L. Romashev, and V. Ustinov, Phys. Rev. Lett. 89, 167203 (2002).

${ }^{17}$ A. Paul, D. E. Bürgler, and P. Grünberg, J. Magn. Magn. Mater. (to be published).

${ }^{18}$ S. K. Sinha, E. B. Sirota, S. Garoff, and H. B. Stanley, Phys. Rev. B 38, 2297 (1988).

${ }^{19}$ A. Paul, T. Damm, D. E. Bürgler, S. Stein, H. Kohlstedt, and P. Grünberg, J. Phys.: Condens. Matter 15, 2471 (2003).

${ }^{20}$ C. Liu, C. Yu, H. Jiang, L. Shen, C. Alexander, and G. J. Mankey, J. Appl. Phys. 87, 6644 (2000).

${ }^{21}$ P. Miltényi, M. Gierlings, J. Keller, B. Beschoten, G. Günterhodt, U. Nowak, and K. D. Usadel, Phys. Rev. Lett. 84, 4224 (2000).

${ }^{22}$ U. Nowak, K. D. Usadel, J. Keller, P. Miltényi, B. Beschoten, and G. Güntherodt, Phys. Rev. B 66, 014430 (2002).

${ }^{23}$ H. Ohldag, A. Scholl, F. Nolting, E. Arenholz, S. Maat, A. T. Young, M. Carey, and J. Stöhr, Phys. Rev. Lett. 91, 017203 (2003).

${ }^{24}$ L. G. Paratt, Phys. Rev. 95, 359 (1954).

${ }^{25}$ H. Zabel, Appl. Phys. A: Solids Surf. 58, 259 (1994).

${ }^{26}$ E. Kentzinger, U. Rücker, and B. P. Toperverg, Physica B 335, 82 (2003).

${ }^{27}$ B. Beckmann, U. Nowak, and K. D. Usadel, Phys. Rev. Lett. 91, 187201 (2003). 